\title{
Plants, clouds and climate
}

\author{
Plant-cloud interactions have the potential to both cool and warm the climate. Ascertaining how these \\ processes balance out at the global scale will require close collaboration between climate scientists and \\ plant biologists.
}

When studying the Earth's climate, biology, chemistry and physics cannot be separated. The interaction between terrestrial plants and the surrounding atmosphere is a case in point. Transpiration from land vegetation dominates terrestrial water fluxes (Nature 496, 347-350; 2013) and land ecosystems take up and sequester large amounts of atmospheric carbon dioxide (Proc. Natl Acad. Sci. USA 104, 18866-18870; 2007). But land plants also exert more subtle effects on the climate: they release large quantities of organic vapour into the atmosphere that, upon oxidation, yields aerosol particles that affect cloud properties (Nature Geosci. 5, 453-458; 2012).

Two studies in this issue suggest that the climatic significance of these biogenic emissions may be greater than thought. A Letter on page 443 shows that the number of cloud droplets is not only affected by aerosols in particulate form, but also by semivolatile organic compounds. These vaporous compounds contribute to droplet growth directly by condensing on growing particles and facilitating further water uptake. The process could have significant consequences for the reflectivity of clouds.

Regardless of the pathway from leaf to cloud, organic vapours seem to lead to more droplets and brighter clouds - a process that could intensify on warming, according to another Letter on page 438 . Here, it is shown that the number of particles in the lower atmosphere large enough to serve as seeds for cloud droplets rises exponentially with temperature, which the authors attribute to growing vapour emissions from plants in warmer conditions. If this plant-driven increase in the atmospheric aerosol burden translates into an increase in the number of cloud droplets as expected, a negative feedback ensues between the terrestrial biosphere and climate warming.

However, it has also been suggested that a reduction in the plant-mediated transfer of water to the atmosphere under higher atmospheric carbon dioxide concentrations could lead to a reduction in water vapour levels in the lower atmosphere (Nature Geosci. 5, 701-704; 2012). The resultant suppression of cloud formation could enhance warming.

How these two parallel but mutually counteracting feedback loops balance out at a global scale will be difficult to quantify, given the regional nature of many of the processes involved. There is clearly much to learn about how plants affect clouds - mediated by water vapour, organic vapours and aerosol particles - in a changing climate.

\section{Double-blind peer review}

\section{Allowing authors of research papers to be anonymous to referees has long been recommended. We will offer such an option, as a trial, from 10 June 2013.}

Nine months ago we promised to look into trialling double-blind peer review as a choice for authors (Nature Geosci. 5, 585; 2012). We are now pleased to announce implementation: Nature Geoscience and Nature Climate Change will allow authors to choose to remain anonymous to referees, just as referees usually remain anonymous to authors.

But will they indeed be anonymous? Perhaps the most popular argument against a double-blind process is that referees will guess the authors' identities, regardless of whether or not the names are actually sent along with the paper, because the content will give them away. We realise that this may be the case occasionally, and we will rely on participating authors to phrase their paper carefully so as to avoid easy identification. Nevertheless, we are convinced that for many papers the double-blind process will serve to remove unconscious biases.

From our experience, authors who try to guess the identity of a referee are very often wrong. It seems unlikely that referees will be any more successful when guessing the identity of authors. Of course, these hunches are usually not verified or falsified, given our obligation to maintain confidentiality. So there is a widespread (and only too human) overconfidence in the accuracy of one's own assumptions. We strongly recommend that referees who receive a paper within the trial do not ponder who the authors might be; authorship should not, in any case, affect their report.

But even if referees correctly identify the research group that a paper is coming from, they are much less likely to guess who the first author is. One of our motivations for setting up a double-blind trial is the possibility that female authors are subjected to tougher peer review than their male colleagues - a distinct possibility in view of evidence that subtle gender biases affect assessments of competence, appropriate salaries and other aspects of academic life (Proc. Natl Acad. Sci. USA 109, 16474-16479;
2012). If the first author is unknown, this bias will be largely removed.

In a reader survey last year (Nature Geosci. $5,585 ; 2012)$, three-quarters of respondents were supportive of double-blind peer review, with only $16 \%$ unconvinced. Interestingly, those who might benefit did not preferentially support a double-blind process: the ratios of males to females, established scientists to young researchers, and people from western countries to scientists elsewhere in the world, were all very similar (down to a per cent or so) between supporters of double-blind peer review and the entire group of respondents.

Determining the impact of a double-blind process on publication outcomes for specific groups of authors will require a larger sample than we will have for a long time. But we will closely monitor uptake of the new doubleblind option, as well as the perceptions of participating authors and referees. We are looking forward to finding out if the option to stay anonymous as an author will prove as popular as the reader survey suggested. 